\title{
Land Suitability Evaluation for Legume Crops and Horticulture Crops in Paman Kallur-1 Micro-Watershed using Geospatial Techniques
}

\author{
N. L. Rajesh ${ }^{1}$, Kirana Kumara ${ }^{1 *}$, V. Rajesh ${ }^{1}$, Chandralekha $^{2}$, Ambika Bhandari $^{2}$, \\ S. Deepika ${ }^{2}$, Basavajyoti ${ }^{2}$, Ambika ${ }^{2}$, H. V. Rudramurthy ${ }^{1}$, K. Basavaraj ${ }^{1}$, \\ U. Satishkumar ${ }^{3}$ and B. K. Desai ${ }^{4}$ \\ ${ }^{1}$ Department of Soil Science, UAS Raichur, Karnataka, India, \\ ${ }^{2}$ Sujala-III, RS \& GIS Lab, UAS, Raichur, India \\ ${ }^{3}$ Department of SWE, CAE, UAS, Raichur, Karnataka, India \\ ${ }^{4}$ Department of Agronomy, Directorate of Research, UAS, Raichur, Karnataka, India \\ *Corresponding author
}

\section{A B S T R A C T}

\section{Keywords}

Land capability,

Crop suitability,

Geospatial

technology and

Micro-watershed

Article Info

Accepted:

12 November 2019

Available Online:

10 December 2019
A detailed study was conducted to assess the land capability and land suitability of Paman kallur-1 micro-watershed. The micro watershed is located in Lingasugur taluk, Raichur district, Karnataka, India, which lies between the latitude and longitude of $76^{0} 37^{\prime} \mathrm{N}-16^{\circ} 7^{\prime} \mathrm{E}$ and $76^{\circ} 39^{\prime} \mathrm{N}-16^{\circ} 5^{\prime} \mathrm{E}$. Initially, land resource survey was carried out at 1:8000 scale to derive soil phase units based on land surface and profile characters. Four soil series were identified and further mapped into six soil phase units. The soil phase unit "THDhD2g1S1R1" covered maximum area of 137 ha (32.22\%) with moderate slope (5-10\%) and moderate erosion in Paman kallur-1 micro-watershed. Land capability with subclasses in the study area was IIIes having limitations of soil erosion, texture, soil drainage, soil fertility and topography. Therefore, 97.15 per cent of the study area is suitable for legume and horticultural purposes. Furthur, Soil phase unit wise and with corresponding survey number, crop plan with suitable interventions for field crops, horticultural crops, vegetables, millets and pulses were prepared for the benefit of the farmers.

\section{Introduction}

The balance between economic viability and destruction of a nation often depends on how the land resource base is managed. Proper land management cannot be done without land use planning (Geetha et al., 2017). An essential part of land use planning is land evaluation. Land evaluation is the assessment of the potential of land for alternative uses using systematic comparison of the land use requirements (LURs) with land quality/ 
characteristics (Dent and Young, 1981). Land evaluation forges a link between the basic survey of resources and the taking of decision on land use planning and management. It puts at the disposal of users relevant information about land resources that are necessary for planning development and taking management decisions (Kharche and Gaikawad, 1993).

North-Eastern dry zone part of Karnataka is having very hot and semi-arid climate. The rainfall region is very erratic and prone to drought and most of the region is covered with black cotton soil with Pigeon pea, Paddy and Cotton as major crops. Soil which is a natural resource has variability inherent to how the soil formation factors interact within the landscape. However, variability can occur also as a result of cultivation, land use and erosion. Salviano (1996) reported spatial variability in soil attributes as a result of land degradation due to erosion.

The land resources inventory in the micro watershed for various crops is necessary to choose the right crop and suitable variety for the area. In order to asses, a detailed land resource inventory and its evaluation were undertaken using geospatial technology. The geospatial techniques are essential for the investigation of spatial variations of soil and crop parameters across agricultural fields, which can lead to the efficient implementation of site-specific management systems (Najafian et al., 2012).

Assessing the extent and degree of suitability of the land resources in the micro watershed for various crops is necessary to choose the right crop and suitable variety for the area. In this regard, a detailed land resource inventory and its evaluation were undertaken using geospatial technology in Paman kallur-1 micro watershed of North Eastern Dry Zone of Karnataka, to characterize land capability and crop suitability.

\section{Materials and Methods}

Paman Kallur-1 micro-watershed (Chiknagnur sub-watershed, Lingasugur taluk, Raichur district) is located in between $76^{0} 37, \mathrm{~N}$ $16^{\circ} 7^{\prime} \mathrm{E}$ and $76^{\circ} 39^{\prime} \mathrm{N}-16^{\circ} 5^{\prime} \mathrm{E}$, covering an area of about 425.72 ha, bounded by Hirenagnur, Gejjalagata, Paman Kallur and Chikhesrur villages (Fig. 1).

The average rainfall of this region is $335 \mathrm{~mm}$. geologically the study area is characterized by granite and gneiss. The detailed land resource survey (at 1:8000 scale) of the entire microwatershed was carried out in the year 2016, with the help of cadastral map overlaid on IRS LISS-IV merged Cartosat-1 imagery having $2.5 \mathrm{~m}$ spatial resolution (Fig. 2). Rapid traversing was carried out to record soils at varying physiographic position. Based on geology, drainage pattern, surface features, slope characteristics and land use, landforms and soil profile were identified (Soil Survey Staff 1999). Four soil series were identified and further mapped into six soil phase units and their area distribution and description were mapped in Figure 3 and Table 1. Morphological characteristics of different soil are shown in Table 3. These data have been used to evaluate the land capability classification (Sehgal, 1996) and land suitability. For various field crops and horticultural crops based on the soil limitations, climatic regimes and land characteristics the suitable interventions with crop plan were developed by NBSS \& LUP.

\section{Results and Discussion}

\section{Land capability classification}

The classification is based on the inherent soil characteristics, external land features and environmental factors that limit the use of land (Table 2). Based on the susceptibility of soils to erosion (e), soils ( $\mathrm{s})$, topography (t) and 
drainage (d) limitations the study area was classified into different land capability classes. Arable lands that are fit for agriculture were grouped under I to IV and non-arable lands were grouped class VI to VIII. Soil morphological characteristics of soil units are matched with land capability classification (Sehgal, 1996).

Based on soil properties, the soils of Paman kallur-1 micro-watershed of Lingasugur taluk have been classified into only one land capability class i.e., IIIes (Fig. 4). All the series of micro-watershed was grouped under class III which is moderately good cultivable lands. These soils were marginally cultivable lands due to severe limitations of erosion, Slope, texture, soil depth limitations. The entire micro-watershed area was fall under IIIes of about 414 ha. Similar findings were also reported by (Leelavathi et al., 2009).

\section{Land suitability classification for crops}

Land suitability was evaluated following FAO (1979) guidelines. It involved formulation of climate and soil requirements of the crop and ratings of these parameters highly suitable (S1), moderately suitable (S2), marginally suitable (S3), and unsuitable (N1). The suitability criteria for sorghum, cotton, tomato and coconut are given by Naidu et al., (2006) was followed and was given in Table 2.

\section{Land suitability for Horticultural crops}

The suitability assessment for horticultural crops in Paman kallur-1 MWS showed that an area of 60 ha $(14.10 \%)$ was moderately suitable (S2) and 354 ha $(83.06 \%)$ was marginally suitable (S3) for Custard Apple with limitations of rooting condition, slope, texture and gravel (Fig. 10). Sapota, Jamun, Guava, Jackfruit, Musambi and Pomegranate covering an area of 197 ha (46.32\%) was marginally suitable and 216 ha $(50.83 \%)$ was currently not suitable with the limitations of rooting condition, slope, texture and gravel (Fig. 11, 12, 14, 15, 17 and 18). Mango and Tamarind tree crops were found to be currently not suitable (N1) due to severe limitations of rooting condition slope, texture and gravel (Fig. 13 and 16, respectively).

\section{Land suitability for legume crops}

The suitability assessment for Pulse crops in Paman kallur-1 MWS showed that an area of 393 ha $(90.05 \%)$ was marginally suitable (S3) and 30 ha $(7.11 \%)$ was currently not suitable (N1) for Ground nut with limitations of rooting condition, texture, slope and gravel (Fig. 5).

In case of Green gram, Bengal gram and Black gram an area of 246 ha $(57.83 \%)$ was moderately suitable, 137 ha $(32.22 \%)$ area was marginally suitable and $30 \mathrm{ha}(7.11 \%)$ area was found to be currently not suitable (N1) due to severe limitations of rooting condition slope, texture and gravel (Fig. 6, 7 and 8 respectively).

Redgram covering an area of 60 ha (14.10\%) was moderately suitable, 323 ha $(75.95 \%)$ was marginally suitable and 30 ha $(7.11 \%)$ area was found to be currently not suitable (N1) due to severe limitations of rooting condition slope, texture and gravel (Fig. 9). Similar findings were also reported by (Rajesh et al., 2018).

\section{Proposed crop plan}

Crop plan for field crops and horticulture crops for HEGmC2 soil phase unit has suitable interventions such as, Deep and wider size pit, Drip irrigation with suitable soil and water conservation measures Cultivation on raised beds with mulches, Graded bunds and strengthening of field bunds for vegetables and field crops. 
Table.1 Mapping units description of Paman kallur-1 micro-watershed

\begin{tabular}{|c|c|c|c|}
\hline Sl. No. & Map symbol & Description & ha $(\%)$ \\
\hline 1 & $\mathrm{CHRhC} 2 \mathrm{~g} 1 \mathrm{~S} 1$ & $\begin{array}{l}\text { Chatra series, Shallow }(25-50 \mathrm{~cm}) \text {, Gently sloping }(1- \\
3 \%) \text {, Sandy Clay loam, textured soil with moderate } \\
\text { erosion, Gravelly }(15-35 \%) \text {, Strong }(0.01 \text { to } 0.1 \%) \text {. }\end{array}$ & $\begin{array}{c}60 \\
(14.08)\end{array}$ \\
\hline 2 & CHRhD2g1S1R1 & $\begin{array}{l}\text { Chatra series, Very shallow }(>25 \mathrm{~cm}) \text {, Moderately sloping } \\
(5-10 \%) \text {, Sandy Clay loam, textured soil with moderate } \\
\text { erosion, Gravelly }(15-35 \%) \text {, Strong }(0.01 \text { to } 0.1 \%) \text {, Fairly } \\
\text { rocky }(2-10 \%) \text {. }\end{array}$ & $\begin{array}{c}49 \\
(11.46)\end{array}$ \\
\hline 3 & CHRhE2g1S1R1 & $\begin{array}{c}\text { Chatra series, Very shallow }(>25 \mathrm{~cm}) \text {, Strongly sloping } \\
(10-15 \%) \text {, Sandy Clay loam, textured soil with moderate } \\
\text { erosion, Gravelly }(15-35 \%) \text {, Strong }(0.01 \text { to } 0.1 \%) \text {, Fairly } \\
\text { rocky }(2-10 \%) \text {. }\end{array}$ & $\begin{array}{c}30 \\
(7.11)\end{array}$ \\
\hline 4 & HEGmC2 & $\begin{array}{l}\text { Heggapur series, Moderately shallow }(50-75 \mathrm{~cm}) \text {, Gently } \\
\text { sloping }(1-3 \%) \text {, Clay, textured soil with moderate erosion. }\end{array}$ & $\begin{array}{c}60 \\
(14.1)\end{array}$ \\
\hline 5 & KMThC2g1S1 & $\begin{array}{c}\text { KumarKhed Tanda series, Shallow }(25-50 \mathrm{~cm}), \text { Gently } \\
\text { sloping(1-3\%), Sandy Clay loam, textured soil with } \\
\text { moderate erosion, erosion, Gravelly }(15-35 \%) \text {, Strong } \\
(0.01 \text { to } 0.1 \%) .\end{array}$ & $\begin{array}{c}77 \\
(18.19)\end{array}$ \\
\hline 6 & THDhD2g1S1R1 & $\begin{array}{c}\text { Thodki series, Moderately shallow }(50-75 \mathrm{~cm}) \text {, } \\
\text { Moderately sloping }(5-10 \%) \text {, Sandy Clay loam, textured } \\
\text { soil with moderate erosion, Gravelly }(15-35 \%) \text {, Strong } \\
(0.01 \text { to } 0.1 \%) \text {, Fairly rocky }(2-10 \%) .\end{array}$ & $\begin{array}{c}137 \\
(32.22)\end{array}$ \\
\hline 7 & Others* & Water body & $\begin{array}{c}12 \\
(2.85)\end{array}$ \\
\hline 8 & Total & & 425.72 \\
\hline
\end{tabular}

Table.2 Suitability criteria table

\begin{tabular}{|c|c|c|c|c|c|}
\hline Crop requirement & \multicolumn{4}{|c|}{ Rating } \\
\hline Soil -site characteristics & Unit & $\begin{array}{c}\text { Highly } \\
\text { suitable (S1) }\end{array}$ & $\begin{array}{c}\text { Moderately } \\
\text { suitable } \\
\text { (S2) }\end{array}$ & $\begin{array}{c}\text { Marginally } \\
\text { suitable } \\
\text { (S3) }\end{array}$ & $\begin{array}{c}\text { Not suitable } \\
\text { (N) }\end{array}$ \\
\hline Slope & $\%$ & $2-3$ & $3-8$ & $8-15$ & $>15$ \\
\hline Length of Growing period & Days & $>110$ & $90-110$ & $60-90$ & $<60$ \\
\hline Soil drainage & class & $\begin{array}{c}\text { Well to mod. } \\
\text { drained }\end{array}$ & imperfect & $\begin{array}{c}\text { Poorly/excessi } \\
\text { vely }\end{array}$ & V. poorly \\
\hline Soil reaction & $\mathrm{pH}$ & $5.5-7.5$ & $7.6-8.5,4.5-$ & $8.6-9.5,4.0-$ & $<4.0$ \\
\hline Surface soil texture & Class & $\begin{array}{c}\text { L, sil, Sl, Cl, } \\
\text { Sicl, Scl }\end{array}$ & Sic, C, Sc & Ls, S, c $>60 \%$ & $\begin{array}{c}\text { S, fragmental } \\
\text { skeletal }\end{array}$ \\
\hline Soil depth & Cm & $>75$ & $50-75$ & $25-50$ & $<25$ \\
\hline Gravel content & $\%$ vol. & $<15$ & $15-30$ & $30-60$ & $>60$ \\
\hline Salinity (EC) & $\mathrm{dSm}$ & $<1.0$ & $1.0-2.0$ & $2.0-4.0$ & $>4$ \\
\hline Sodicity (ESP) & $\%$ & $<10$ & $10-15$ & $15-25$ & $>25$ \\
\hline
\end{tabular}


Table.3 Physico-chemical properties of soil series

\begin{tabular}{|c|c|c|c|c|c|c|c|c|c|c|}
\hline \multirow[b]{2}{*}{ Series } & \multirow[b]{2}{*}{ Mapping unit } & \multirow[t]{2}{*}{ Geology } & \multirow{2}{*}{$\begin{array}{l}\text { Depth } \\
\text { (cm) }\end{array}$} & \multirow{2}{*}{$\begin{array}{c}\text { Slope } \\
\%\end{array}$} & \multicolumn{2}{|c|}{ Colour } & \multicolumn{2}{|c|}{ Texture } & \multirow[t]{2}{*}{ Land use } & \multirow[t]{2}{*}{ Drainage } \\
\hline & & & & & Surface & Subsurface & Surface & Subsurface & & \\
\hline \multirow[t]{3}{*}{ CHR } & CHRhC2g1S1 & Granite & \multirow[t]{3}{*}{$25-50$} & \multirow[t]{3}{*}{$3-10 \%$} & \multirow{3}{*}{$\begin{array}{c}5 \mathrm{YR} \\
4 / 4,3 / 3\end{array}$} & \multirow{3}{*}{$\begin{array}{c}5 \mathrm{YR} \\
3 / 4,4 / 6\end{array}$} & \multirow{3}{*}{$\begin{array}{l}\text { Sandy clay } \\
\text { loam, sandy } \\
\text { loam }\end{array}$} & \multirow{3}{*}{$\begin{array}{l}\text { Sandy clay } \\
\text { loam, } \\
\text { sandy loam }\end{array}$} & \multirow[t]{3}{*}{ Agriculture } & \multirow[t]{3}{*}{ Well drained } \\
\hline & CHRhD2g1S1R1 & Granite & & & & & & & & \\
\hline & CHRhE2g1S1R1 & Granite & & & & & & & & \\
\hline HEG & HEGmC2 & Gneiss & $50-75$ & $1-5 \%$ & $\begin{array}{c}10 \mathrm{YR} \\
3 / 3,3 / 1\end{array}$ & $\begin{array}{c}10 \mathrm{YR} \\
3 / 3,3 / 2,, 4 / 4\end{array}$ & $\begin{array}{l}\text { Sandy clay } \\
\text { loam }\end{array}$ & $\begin{array}{l}\text { Sandy clay } \\
\text { loam }\end{array}$ & Agriculture & Well drained \\
\hline KMT & KMThC2g1S1 & Gneiss & $25-50$ & $3-10 \%$ & $\begin{array}{l}7.5 \text { YR } \\
4 / 3,5 / 3\end{array}$ & $\begin{array}{l}7.5 \text { YR } \\
4 / 3,3 / 2\end{array}$ & Sandy clay & Sandy Clay & Agriculture & $\begin{array}{l}\text { Moderately } \\
\text { well drained }\end{array}$ \\
\hline THD & THDhD2g1S1R1 & Granite & $50-75$ & $1-5 \%$ & $\begin{array}{c}5 \mathrm{YR} \\
3 / 4,2.5 \\
\mathrm{YR} 5 / 6\end{array}$ & $\begin{array}{c}5 \mathrm{YR} \\
3 / 4,2.5 \mathrm{YR} \\
3 / 4\end{array}$ & $\begin{array}{l}\text { Sandy clay } \\
\text { loam }\end{array}$ & $\begin{array}{l}\text { Sandy clay } \\
\text { loam }\end{array}$ & Agriculture & $\begin{array}{l}\text { Moderately } \\
\text { well drained }\end{array}$ \\
\hline
\end{tabular}

\begin{tabular}{|c|c|c|c|c|c|c|c|c|c|c|}
\hline \multirow[b]{2}{*}{ Series } & \multirow[b]{2}{*}{ Mapping unit } & \multirow[t]{2}{*}{ Rockiness } & \multirow{2}{*}{$\begin{array}{c}\text { Gravelliness } \\
\%\end{array}$} & \multirow[t]{2}{*}{ Stoniness } & \multicolumn{2}{|c|}{ Structure } & \multicolumn{2}{|c|}{ Consistency } & \multirow[t]{2}{*}{ pH } & \multirow[t]{2}{*}{ EC } \\
\hline & & & & & Surface & Subsurface & Surface & Subsurface & & \\
\hline \multirow[t]{3}{*}{ CHR } & CHRhC2g1S1 & nil & \multirow{3}{*}{ Gravelly } & \multirow[t]{3}{*}{ strong } & \multirow[t]{3}{*}{$1 \mathrm{fmsbk}$} & \multirow[t]{3}{*}{2 fmsbk } & \multirow{3}{*}{$\begin{array}{l}\text { sh, fr, ss, } \\
\text { sp }\end{array}$} & \multirow[t]{3}{*}{ h, fr, vs, vp } & \multirow[t]{3}{*}{ Neutral } & \multirow[t]{3}{*}{ Non saline } \\
\hline & CHRhD2g1S1R1 & $\begin{array}{l}\text { Fairly } \\
\text { rocky }\end{array}$ & & & & & & & & \\
\hline & CHRhE2g1S1R1 & $\begin{array}{l}\text { Fairly } \\
\text { rocky }\end{array}$ & & & & & & & & \\
\hline HEG & HEGmC2 & nil & nil & nil & 1ffmgrsbk & 2 fmsbk & $\begin{array}{l}\text { sh, fr, ss, } \\
\text { sp }\end{array}$ & h, fr, vs, vp & $\begin{array}{c}\text { Moderately } \\
\text { alkaline }\end{array}$ & Non saline \\
\hline KMT & KMThC2g1S1 & nil & Gravelly & strong & $1 \mathrm{fmsbk}$ & 2 fmsbk & $\begin{array}{c}\text { sh, fr, ss, } \\
\text { sp }\end{array}$ & $\begin{array}{l}\text { vh, fr, vs, } \\
\text { vp }\end{array}$ & $\begin{array}{l}\text { Slightly } \\
\text { alkaline }\end{array}$ & Non saline \\
\hline THD & THDhD2g1S1R1 & $\begin{array}{l}\text { Fairly } \\
\text { rocky }\end{array}$ & Gravelly & strong & $1 \mathrm{fmsbk}$ & $2 \mathrm{fmsbk}$ & $\begin{array}{l}\text { sh, fr, ss, } \\
\text { sp }\end{array}$ & h, fr, s, vp & $\begin{array}{l}\text { Moderately } \\
\text { alkaline }\end{array}$ & Non saline \\
\hline
\end{tabular}

Note: $\mathrm{sh}$ - slighly hard, fr - friable, ss - slightly sticky, sp - slightly plastic, fi - firm, vs - very sticky, v p - very plastic, $\mathrm{h}$ - hard, $\mathrm{m}$ - medium, sbk - sub angular blocky, abk-angular bloky, gr-granular, $\mathrm{c}$ - coarse, fc-few common roots, f-fine root 
Table.4 Proposed Crop Plan for Paman kallur-1 Micro-watershed

\begin{tabular}{|c|c|c|c|c|c|}
\hline LMU & Mapping unit & Survey number & Field crops & Horticulture crops & Suitable Intervention \\
\hline LMU-1 & $\begin{array}{c}\text { CHRhC2g1S1 } \\
\text { CHRhD2g1S1R1 } \\
\text { CHRhE2g1S1R1 }\end{array}$ & $\begin{array}{c}\text { Hirenagnur:- } \\
\text { 165,70,193,194,198, } \\
\text { 193,210,209,208. } \\
\text { Gejjalagatta:- } \\
\text { 76,73,74,75,3 } \\
\text { Chikhesrur:- } \\
\text { 23,22,65,62,66,61,58 } \\
\text {,35,34,33,30,32,180, } \\
\text { 149,51,151,158. }\end{array}$ & $\begin{array}{c}\text { Sole crop; } \\
\text { sorghum, Bajra, Navni, Red } \\
\text { gram, Green gram, Cotton, } \\
\text { Maize, Sun flower, black gram, } \\
\text { bengal gram, ground nut, } \\
\text { maize }\end{array}$ & $\begin{array}{l}\text { Fruit crops : custard apple, Tamarind, } \\
\text { jamun, Ber, Sapota, Anola, } \\
\text { Veg: Onion, Tomato, Brinjal, Chilli, } \\
\text { Bhendi, Green leaf, cury leaf, Tomato, } \\
\text { Flowers-Gaillardia, marigold, } \\
\text { Chrysanthemum, lilly }\end{array}$ & $\begin{array}{l}\text { Deep and wider size pit, Drip } \\
\text { irrigation with suitable soil and } \\
\text { water conservation measures } \\
\text { Cultivation on raised beds with } \\
\text { mulches and drip. }\end{array}$ \\
\hline LMU-2 & KMThC2g1S1 & $\begin{array}{c}\text { Paman kallur:- } \\
\text { 172,171,170/1,173/2, } \\
\text { 170/2,7,8,169,179,9( } \\
\text { 1),170,177/1,177/2,1 } \\
80 / 1,180 / 1,152,150,1 \\
84 . \\
\text { Hirenagnur:- } \\
\text { 212. } \\
\text { Chikhesrur:- } \\
\text { 27,26,31. }\end{array}$ & $\begin{array}{c}\text { Sole crop; } \\
\text { sorghum, Bajra, Navni, Red } \\
\text { gram, Green gram, Cotton, } \\
\text { Maize, Sun flower, black gram, } \\
\text { bengal gram, ground nut } \\
\text { maize }\end{array}$ & $\begin{array}{l}\text { Fruit crops : custard apple, Tamarind, } \\
\text { jamun, Ber, Sapota, Anola, } \\
\text { Veg: Onion, Tomato, Brinjal, Chilli, } \\
\text { Bhendi, Green leaf, cury leaf, Tomato, } \\
\text { Flowers-Gaillardia, marigold, } \\
\text { Chrysanthemum, lilly }\end{array}$ & $\begin{array}{l}\text { Deep and wider size pit, Drip } \\
\text { irrigation with suitable soil and } \\
\text { water conservation measures } \\
\text { Cultivation on raised beds with } \\
\text { mulches and drip. }\end{array}$ \\
\hline LMU-3 & THDhD2g1S1R1 & $\begin{array}{c}\text { Hirenagnur:- } \\
\text { 84,192,191,200,205, } \\
\text { 206,207,204,203. } \\
\text { Gejjalagatta:- } \\
\text { 4(1),2. }\end{array}$ & $\begin{array}{c}\text { Sole crop; } \\
\text { sorghum, Bajra, Navni, Red } \\
\text { gram, Green gram, Cotton, } \\
\text { Maize, Sun flower, black gram, } \\
\text { bengal gram, ground nut, } \\
\text { maize }\end{array}$ & $\begin{array}{l}\text { Fruit crops : custard apple, Tamarind, } \\
\text { jamun, Ber, Sapota, Anola, } \\
\text { Veg: Onion, Tomato, Brinjal, Chilli, } \\
\text { Bhendi, Green leaf, cury leaf, Tomato, } \\
\text { Flowers-Gaillardia, marigold, } \\
\text { Chrysanthemum, lilly }\end{array}$ & $\begin{array}{l}\text { Deep and wider size pit, Drip } \\
\text { irrigation with suitable soil and } \\
\text { water conservation measures } \\
\text { Cultivation on raised beds with } \\
\text { mulches and drip. }\end{array}$ \\
\hline LMU-4 & HEGmC2 & $\begin{array}{c}\text { Hirenagnur:- } \\
\text { 186/1,189,70,158,15 } \\
5,154 / 2,154 / 3 .\end{array}$ & $\begin{array}{c}\text { Sole crop; } \\
\text { sorghum, Bajra, Navni, Red } \\
\text { gram, Green gram, Cotton, } \\
\text { Maize, Sun flower, black gram, } \\
\text { bengal gram, ground nut, } \\
\text { maize }\end{array}$ & $\begin{array}{l}\text { Fruit crops : Sapota, Jamun, Guava, } \\
\text { Tamarind, Lime, Musambhi, Custard } \\
\text { apple, Jackfruit, Amla, pomegranate, } \\
\text { Veg: Onion, Tomato, Brinjal, Chilli, } \\
\text { Bhendi, Green leaf, curry leaf, Tomato, } \\
\text { Flowers-Gaillardia, marigold, } \\
\text { Chrysanthemum, lilly }\end{array}$ & $\begin{array}{l}\text { Deep and wider size pit, Drip } \\
\text { irrigation with suitable soil and } \\
\text { water conservation measures } \\
\text { Cultivation on raised beds with } \\
\text { mulches and drip. Graded bunds } \\
\text { and strengthening of field bunds }\end{array}$ \\
\hline
\end{tabular}


Fig.1 Location map of Paman kallur-1 micro-watershed

\section{LOCATION MAP OF PAMAN KALLUR-1 MICRO-WATERSHED}
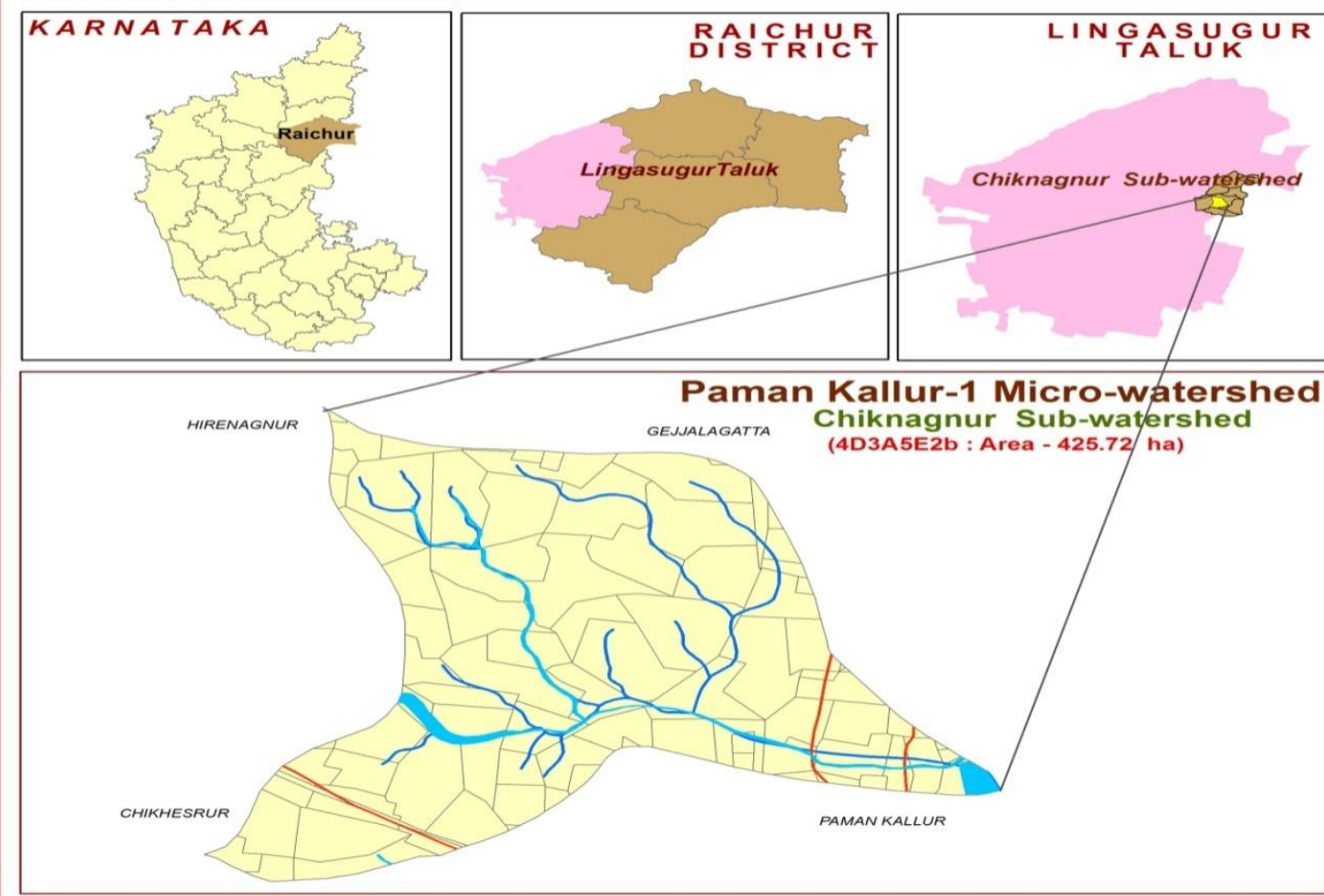

Fig.2 Satellite image of Paman kallur-1 micro-watershed

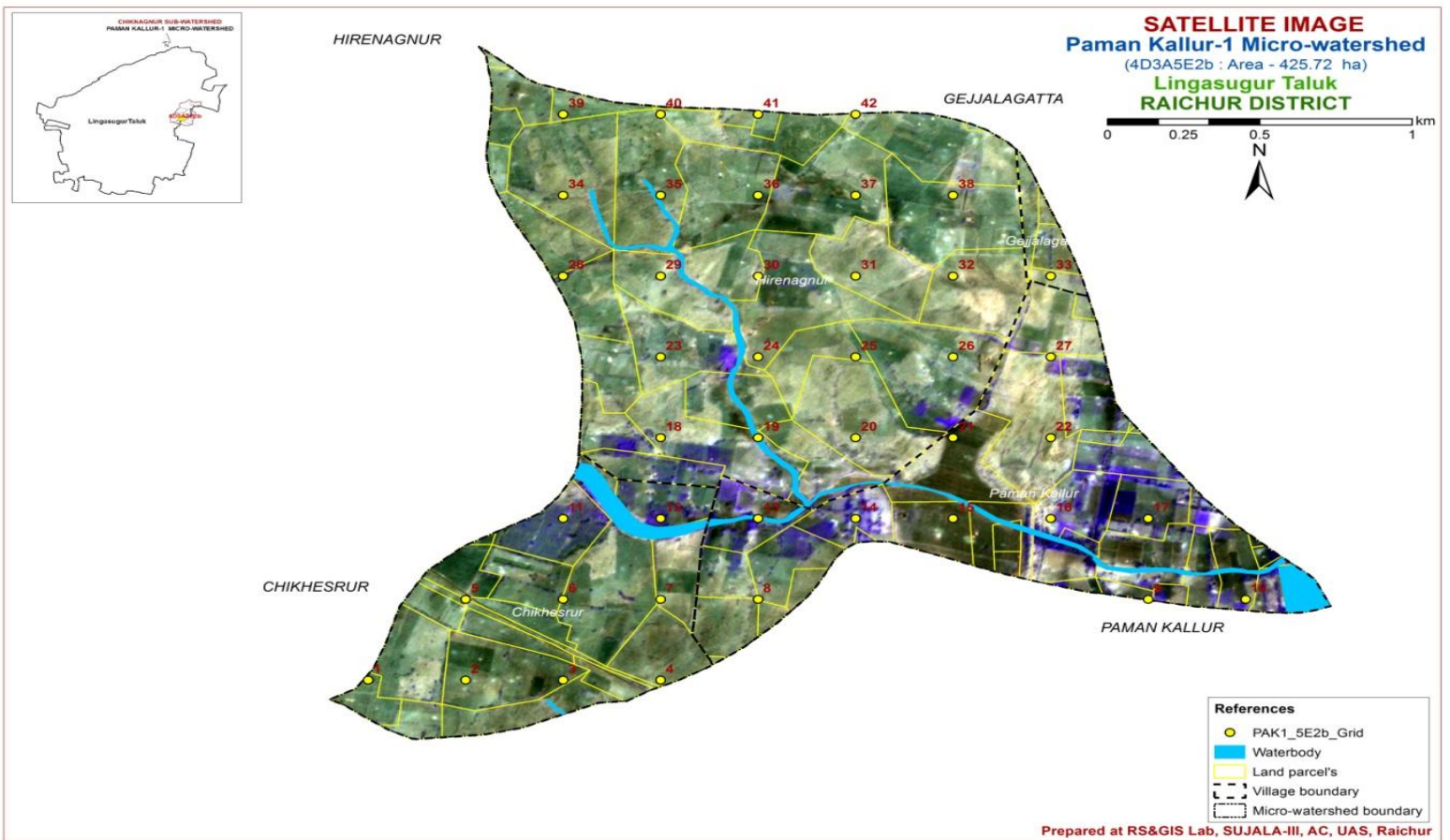


Fig.3 Soil map of Paman kallur-1 micro-watershed

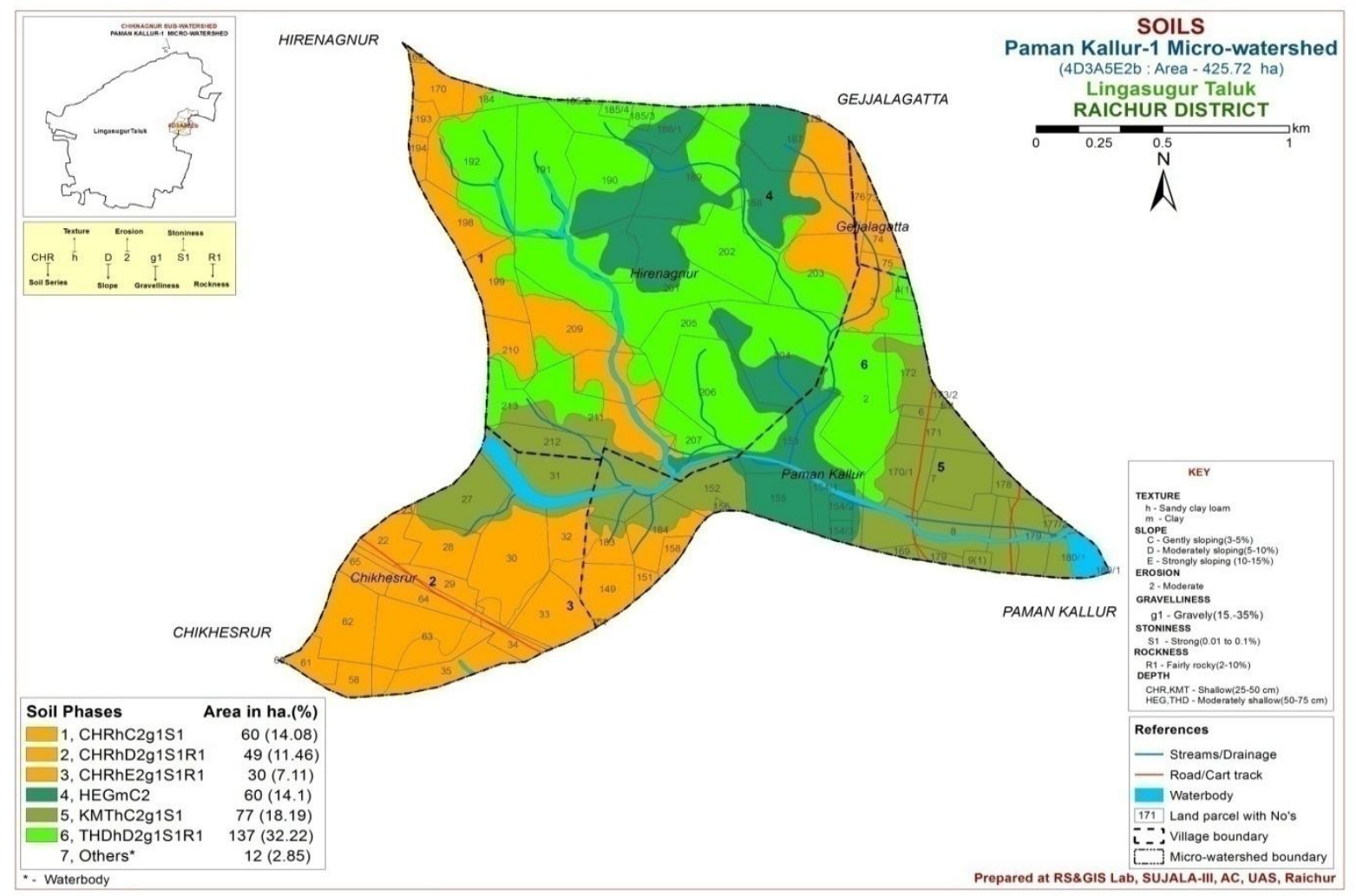

Fig.4 Land capability map of Paman kallur-1 micro-watershed

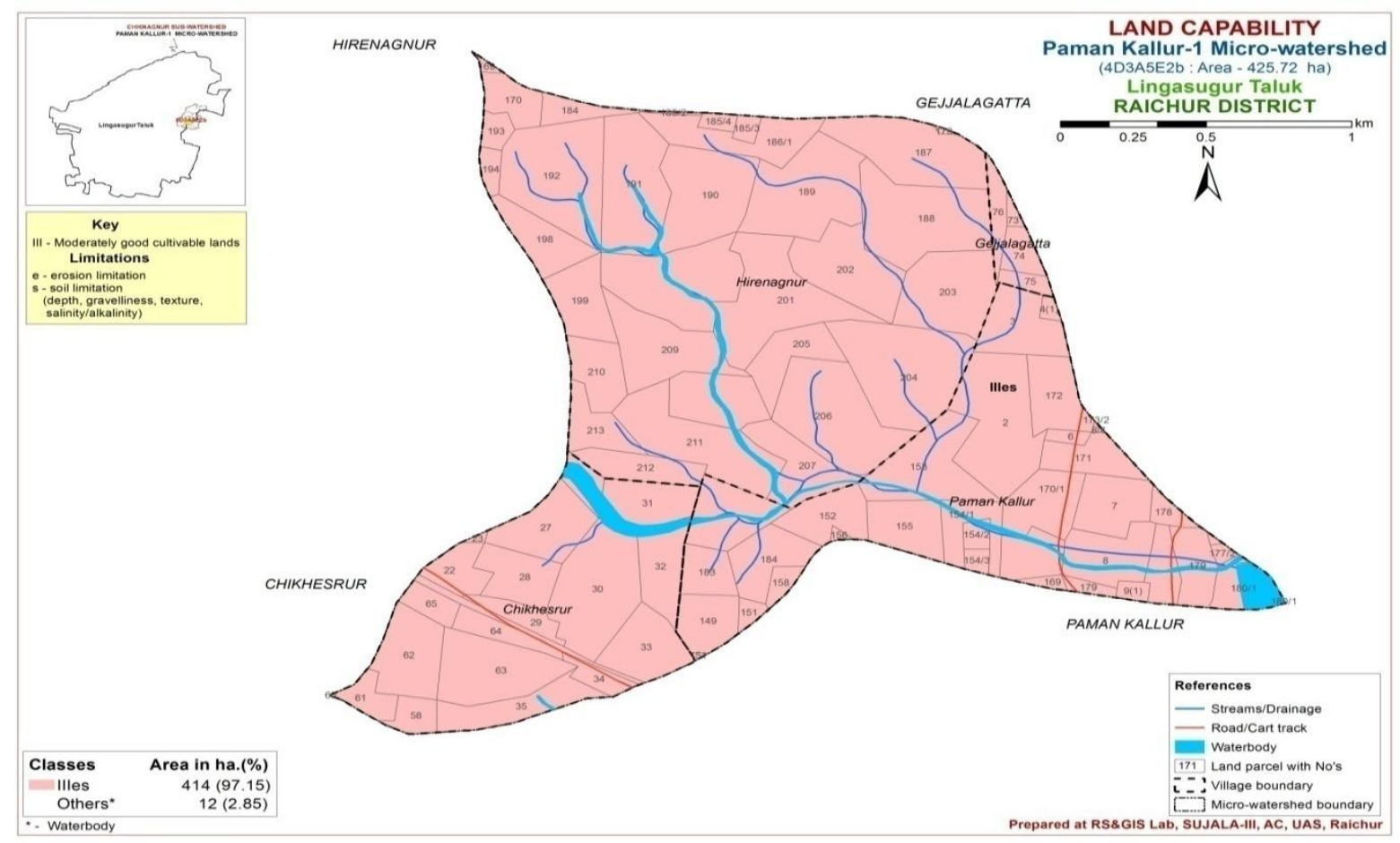


Fig.5 Land suitability map for Ground nut in Paman kallur-1 micro-watershed

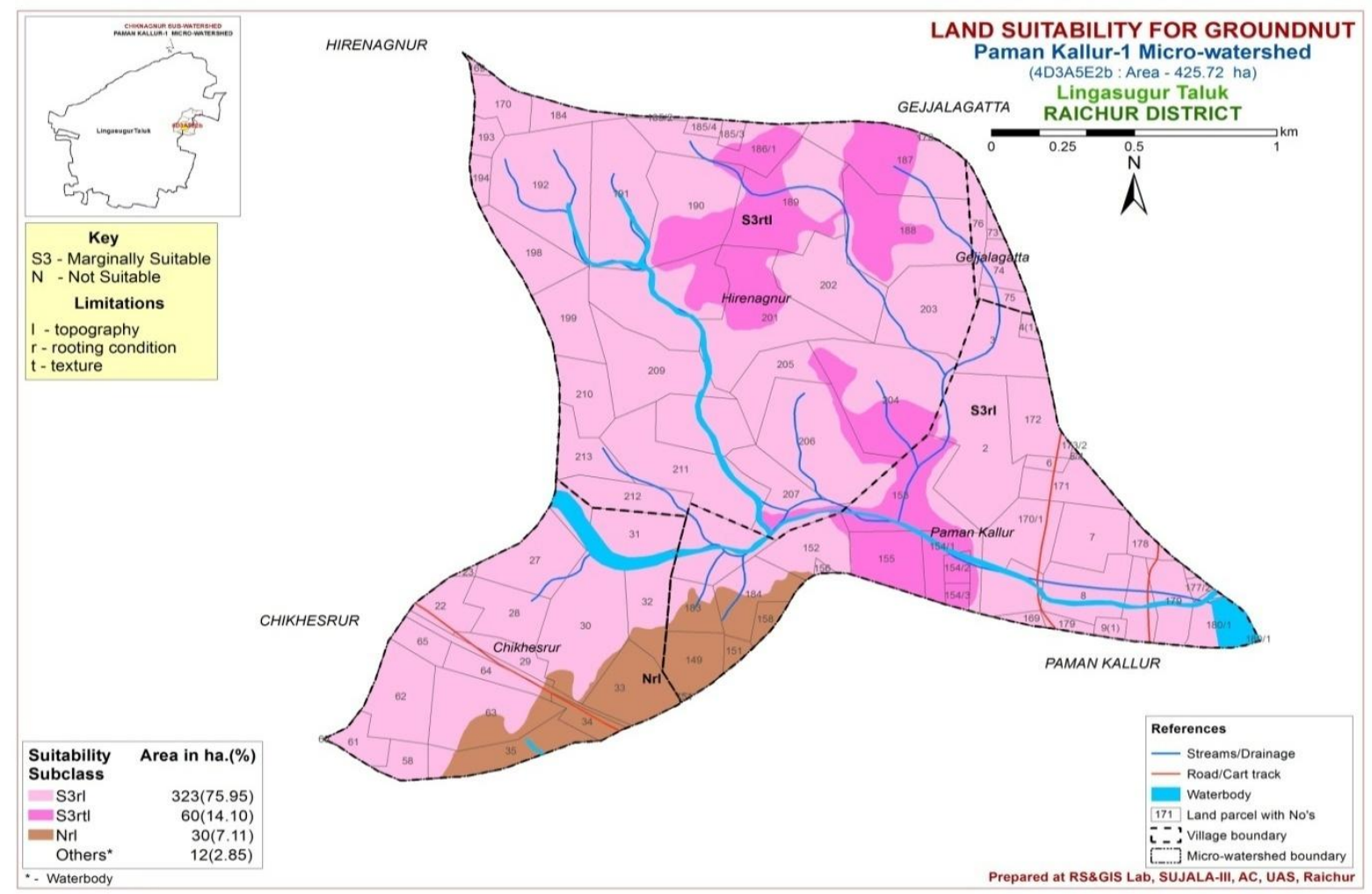

Fig.6 Land suitability map for Green gram in Paman kallur-1 micro-watershed

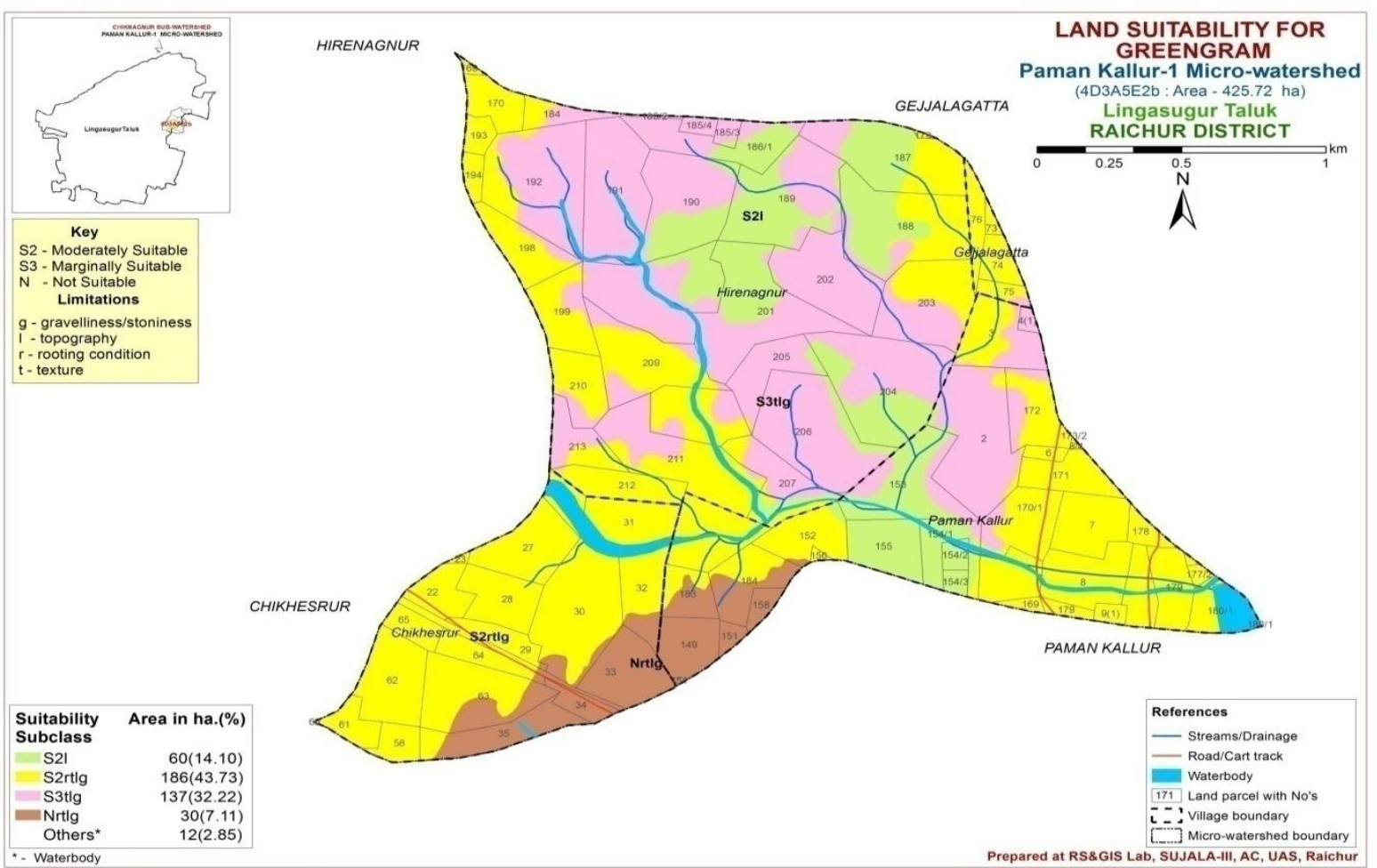


Fig.7 Land suitability map for Bengal gram in Paman kallur-1 micro-watershed

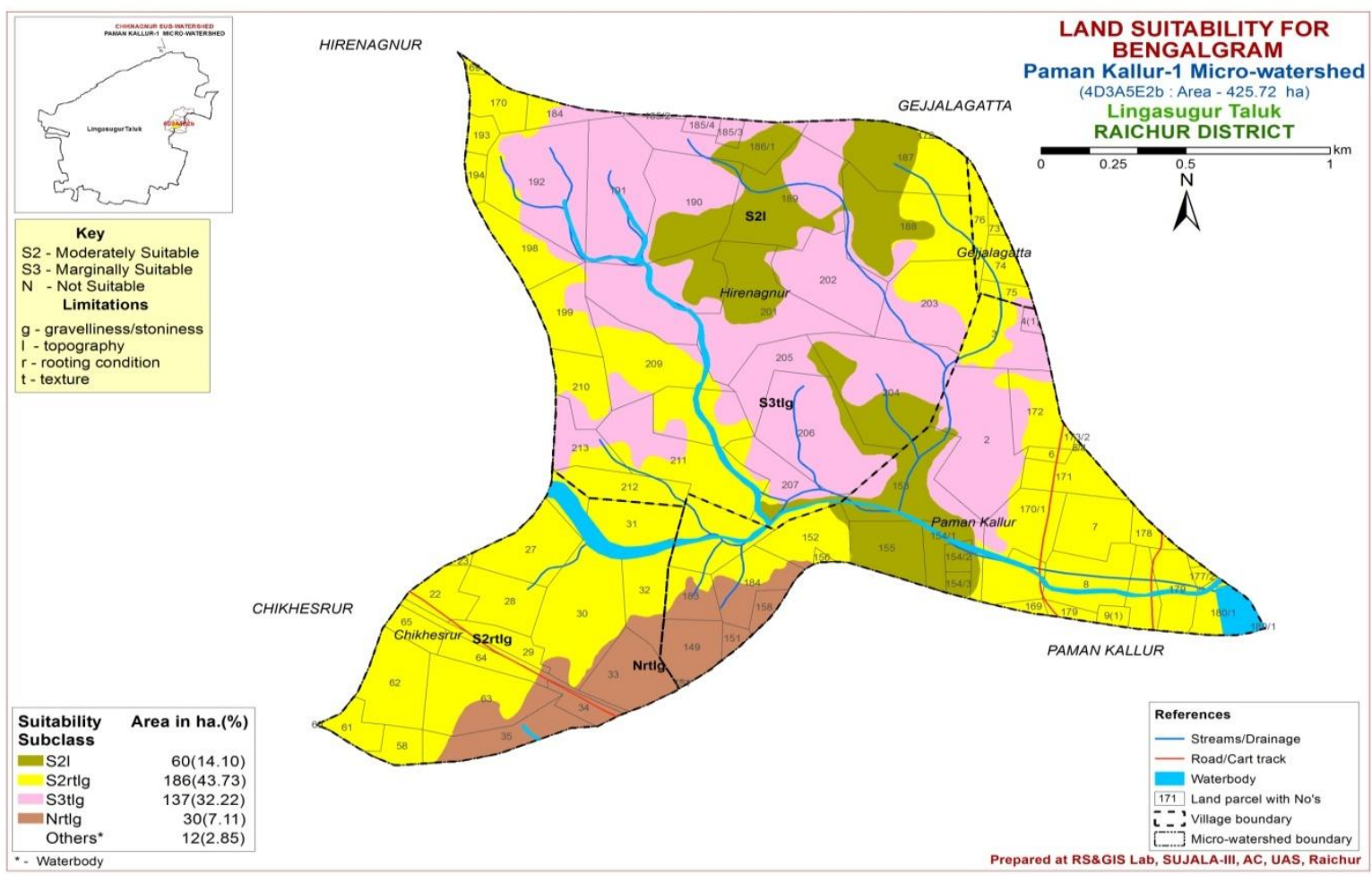

Fig.8 Land suitability map for Black gram in Paman kallur-1 micro-watershed

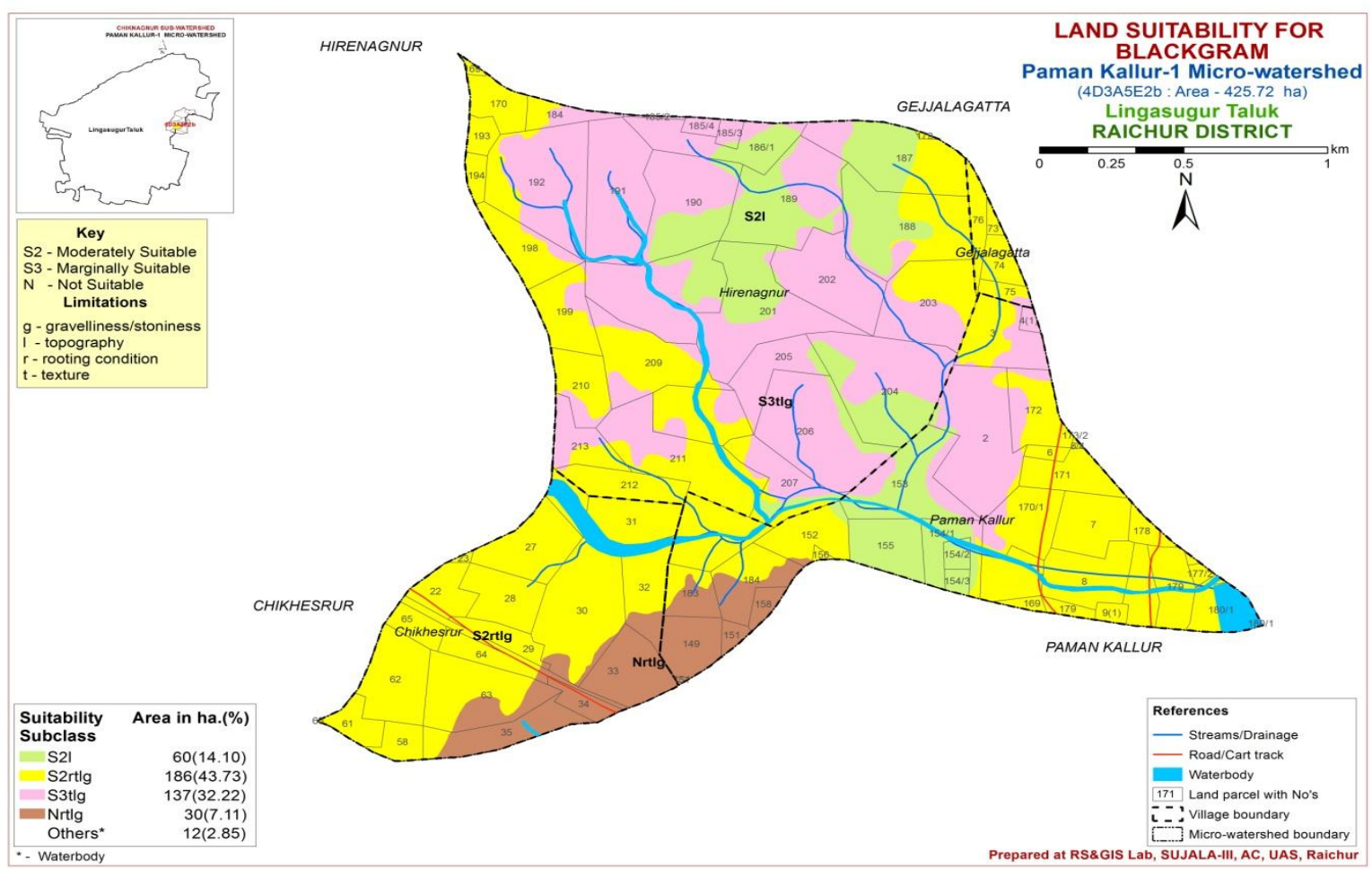


Fig.9 Land suitability map for Redgram in Paman kallur-1 micro-watershed

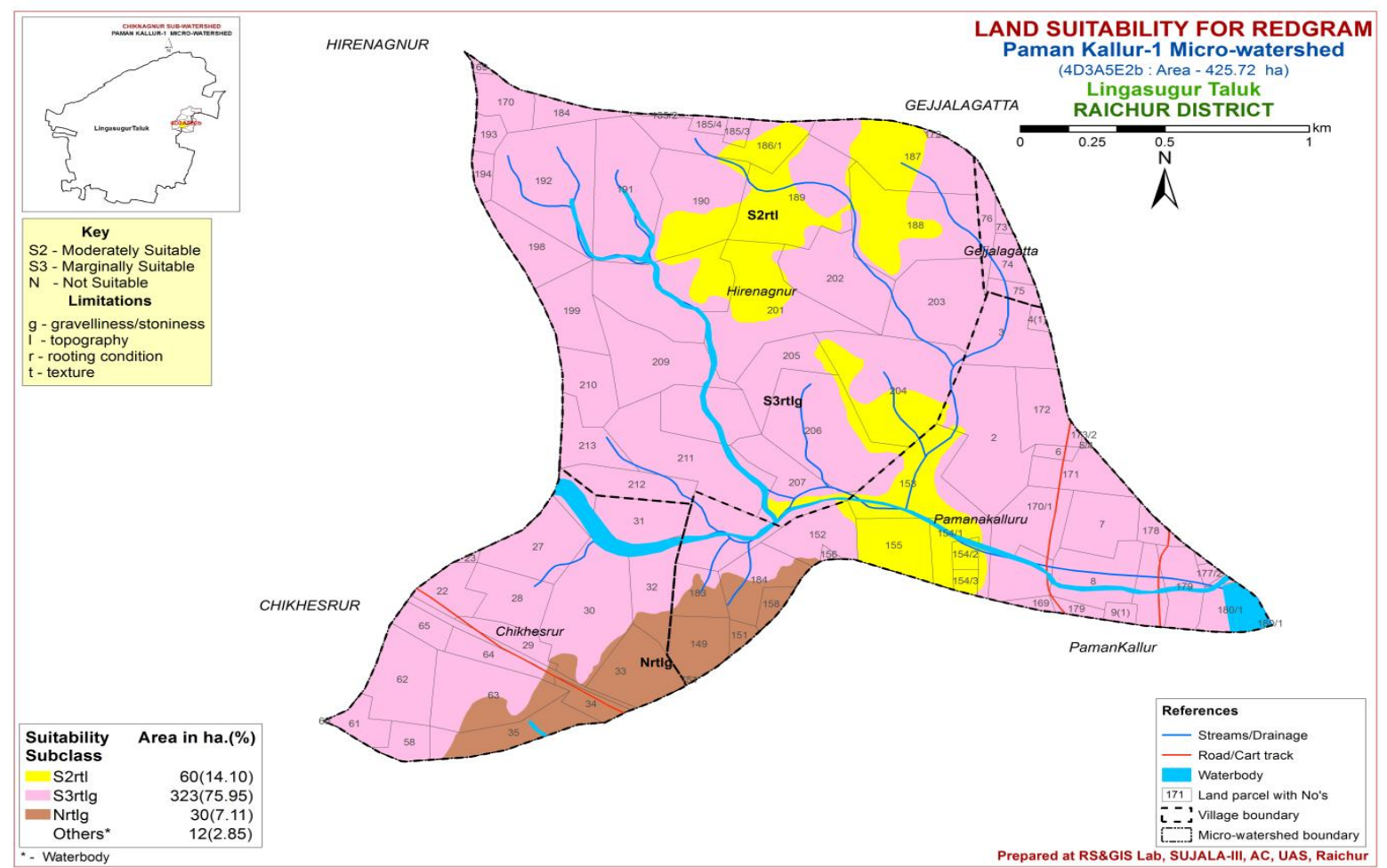

Fig.10 Land suitability map for Custard apple in Paman kallur-1 micro-watershed

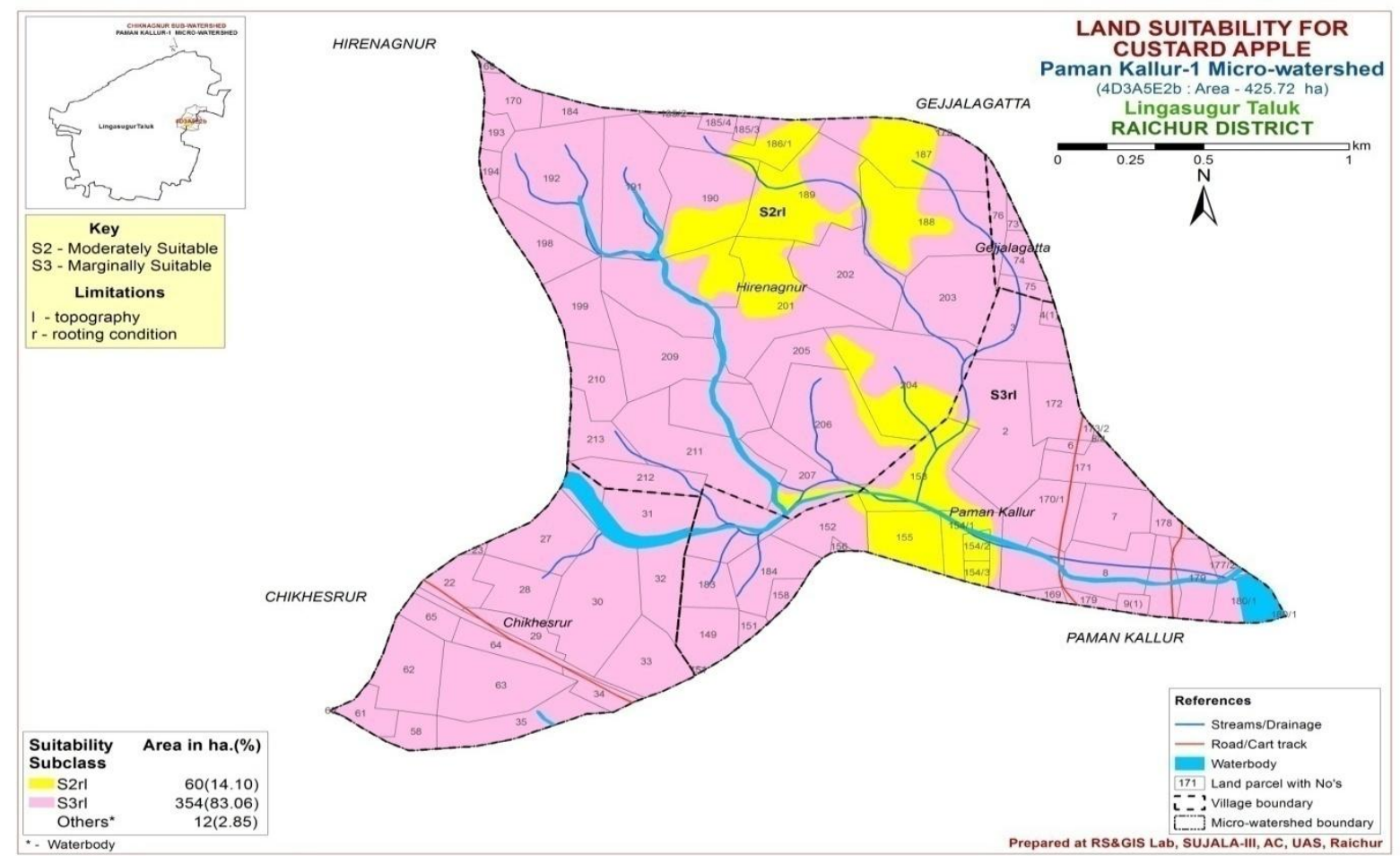


Fig.11 Land suitability map for Guava in Paman kallur-1 micro-watershed

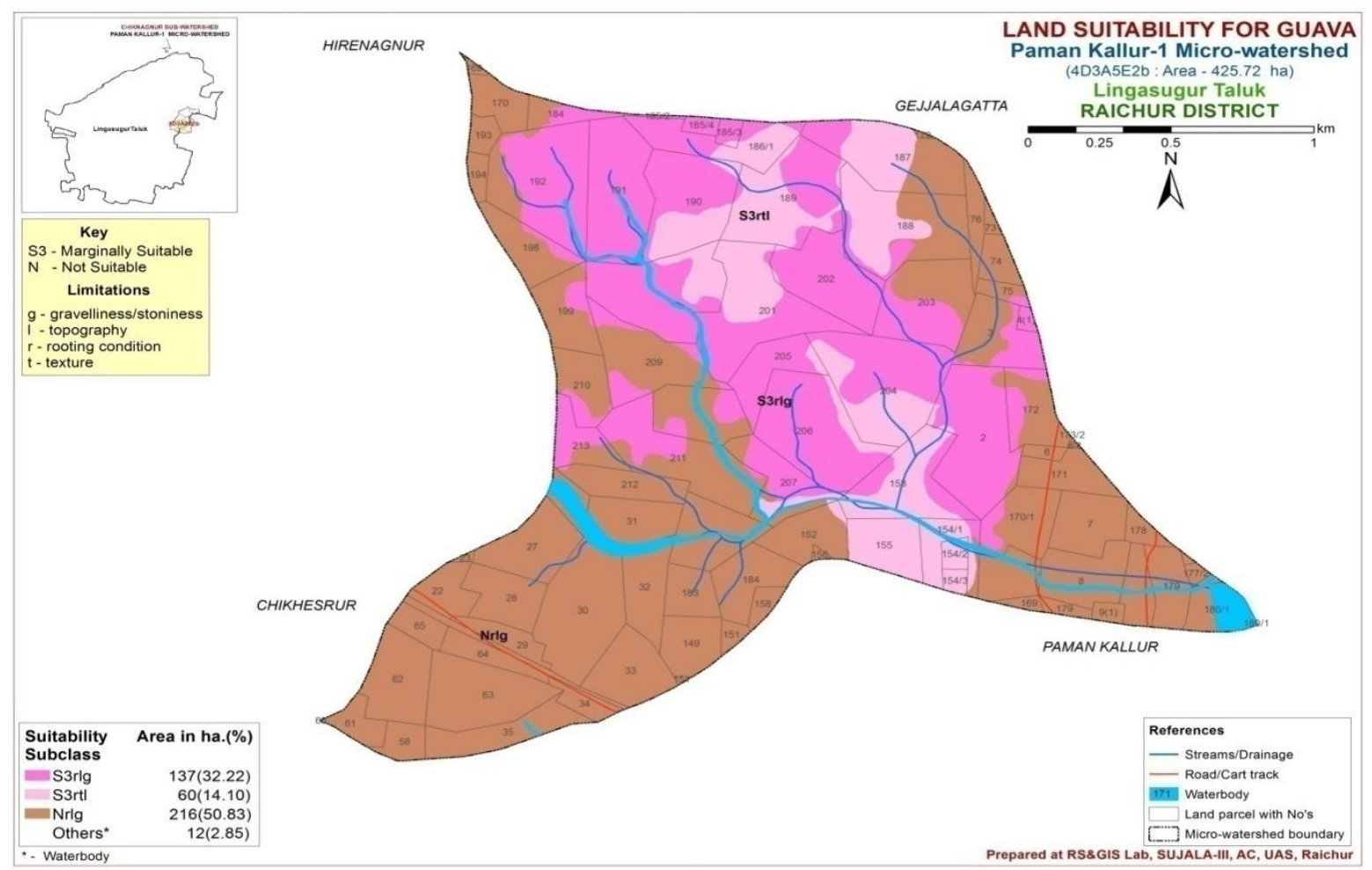

Fig.12 Land suitability map for Jamun in Paman kallur-1 micro-watershed

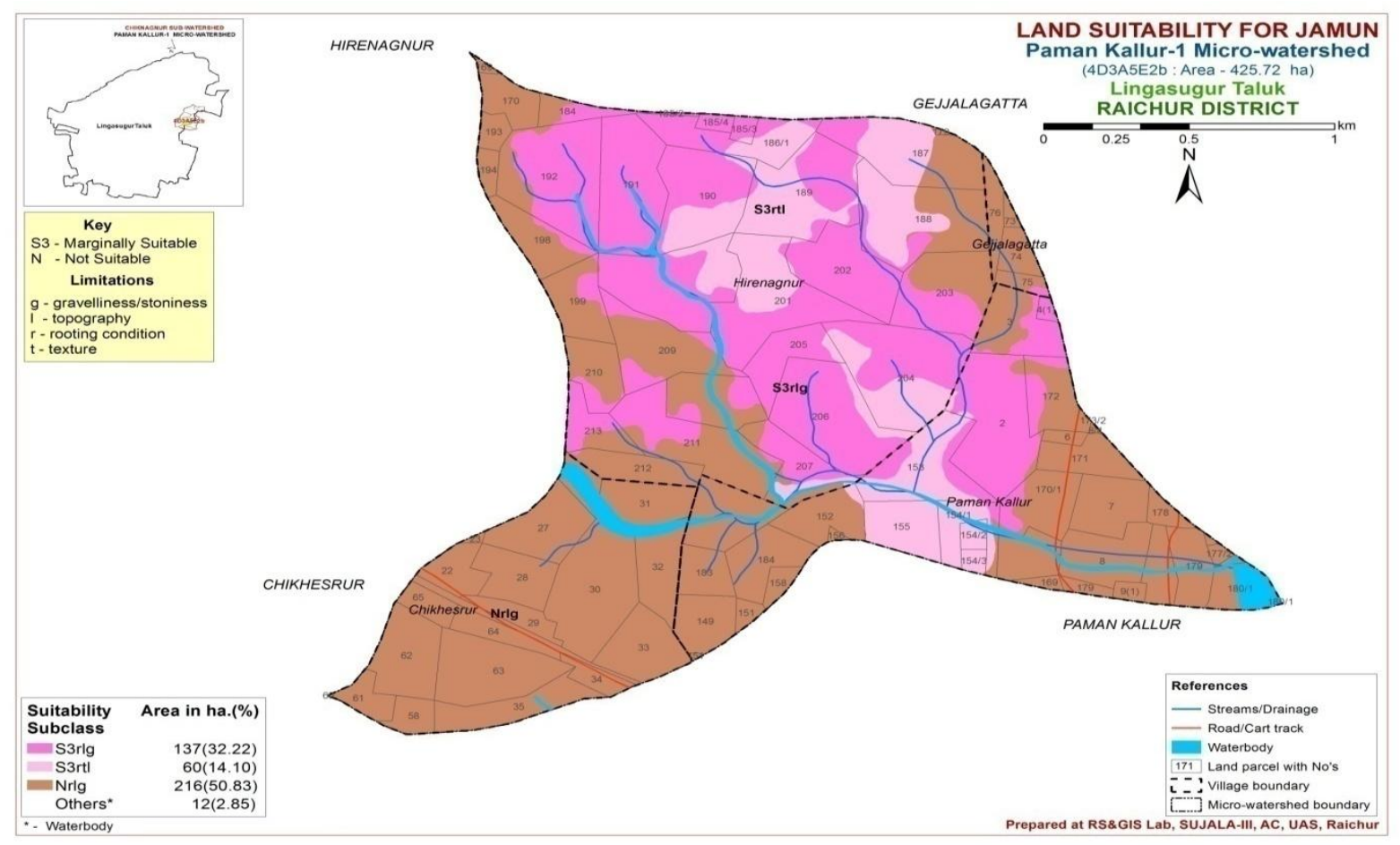


Fig.13 Land suitability map for Mango in Paman kallur-1 micro-watershed

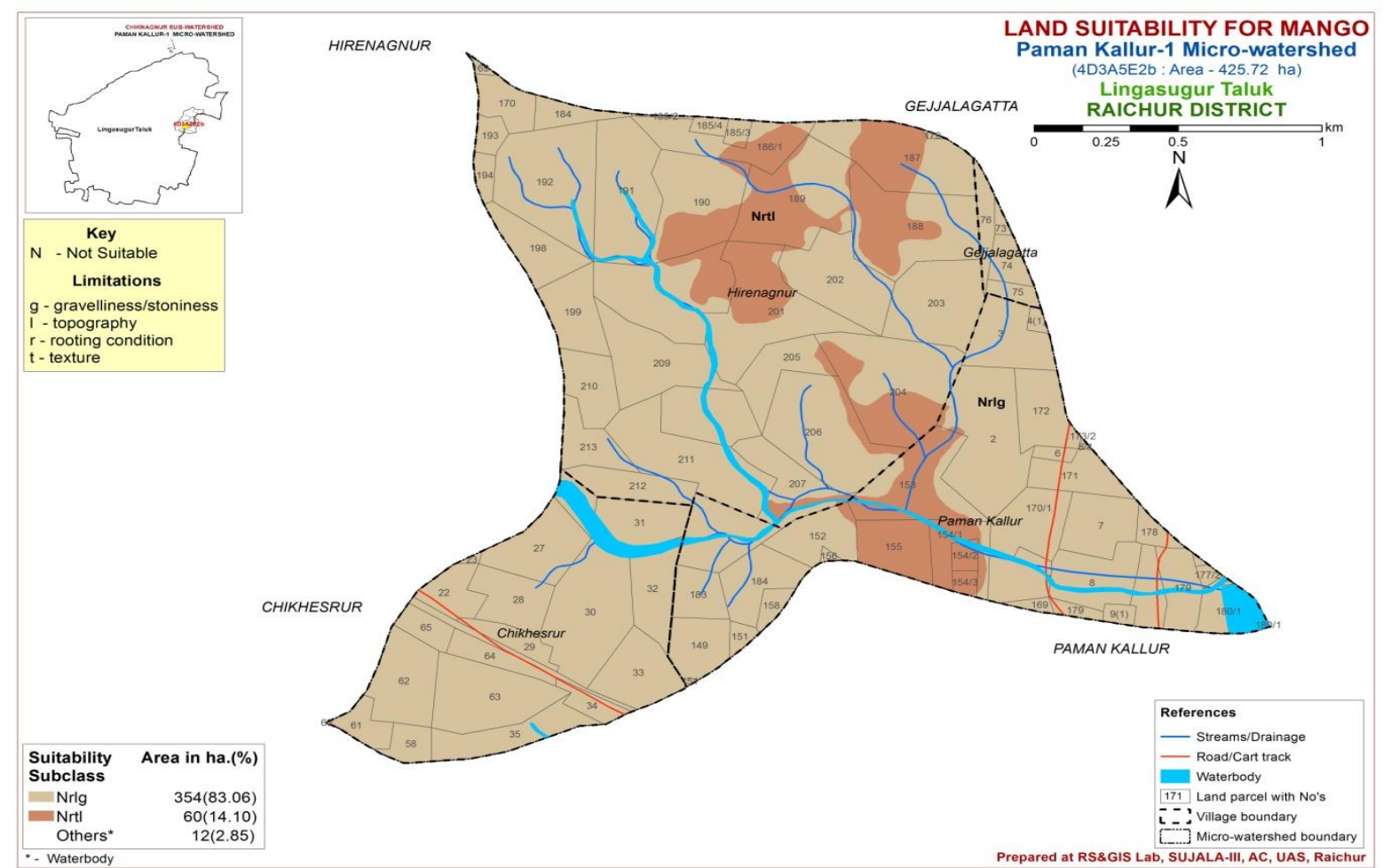

Fig.14 Land suitability map for Sapota in Paman kallur-1 micro-watershed

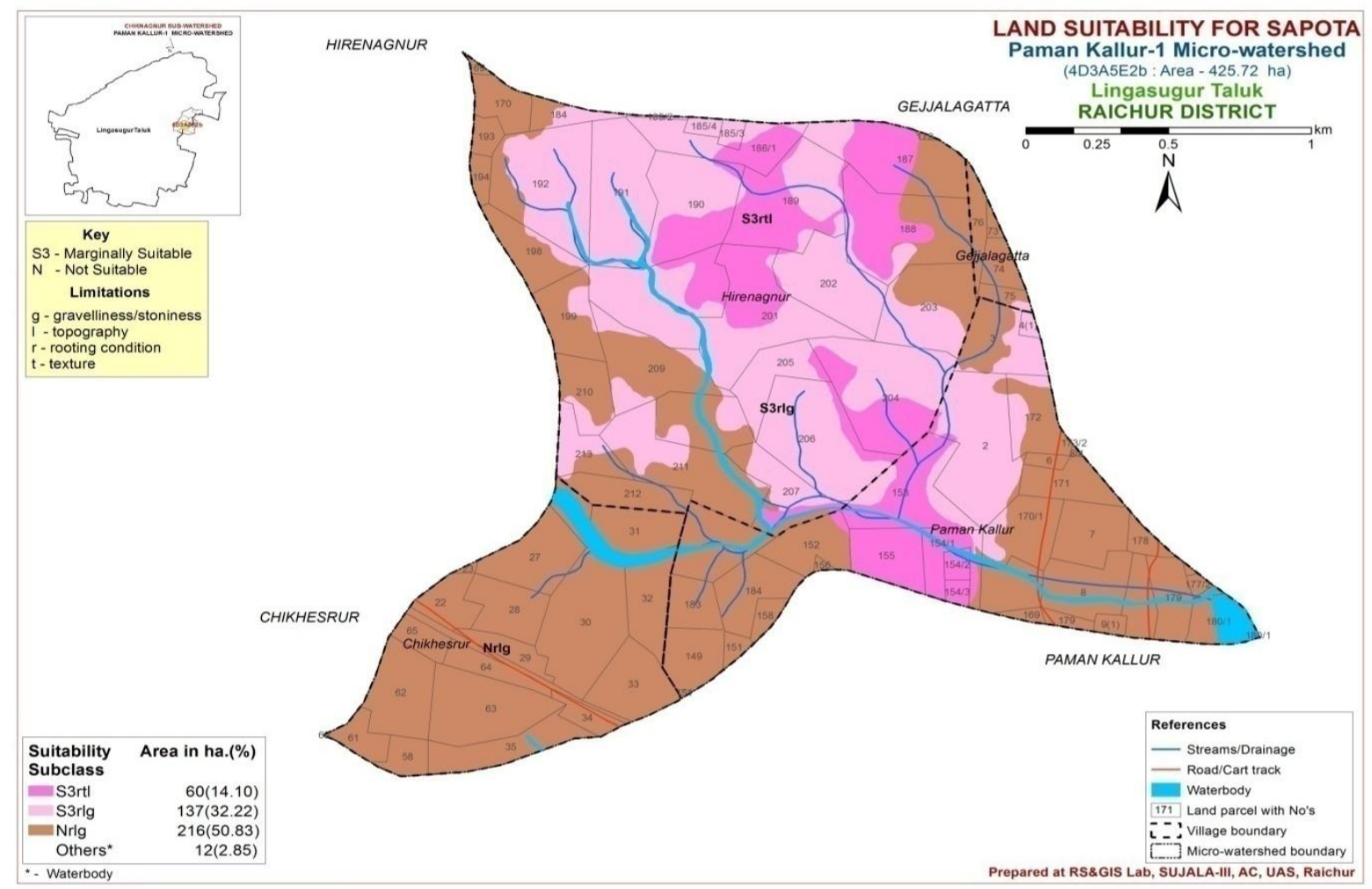


Fig.15 Land suitability map for Musambi in Paman kallur-1 micro-watershed

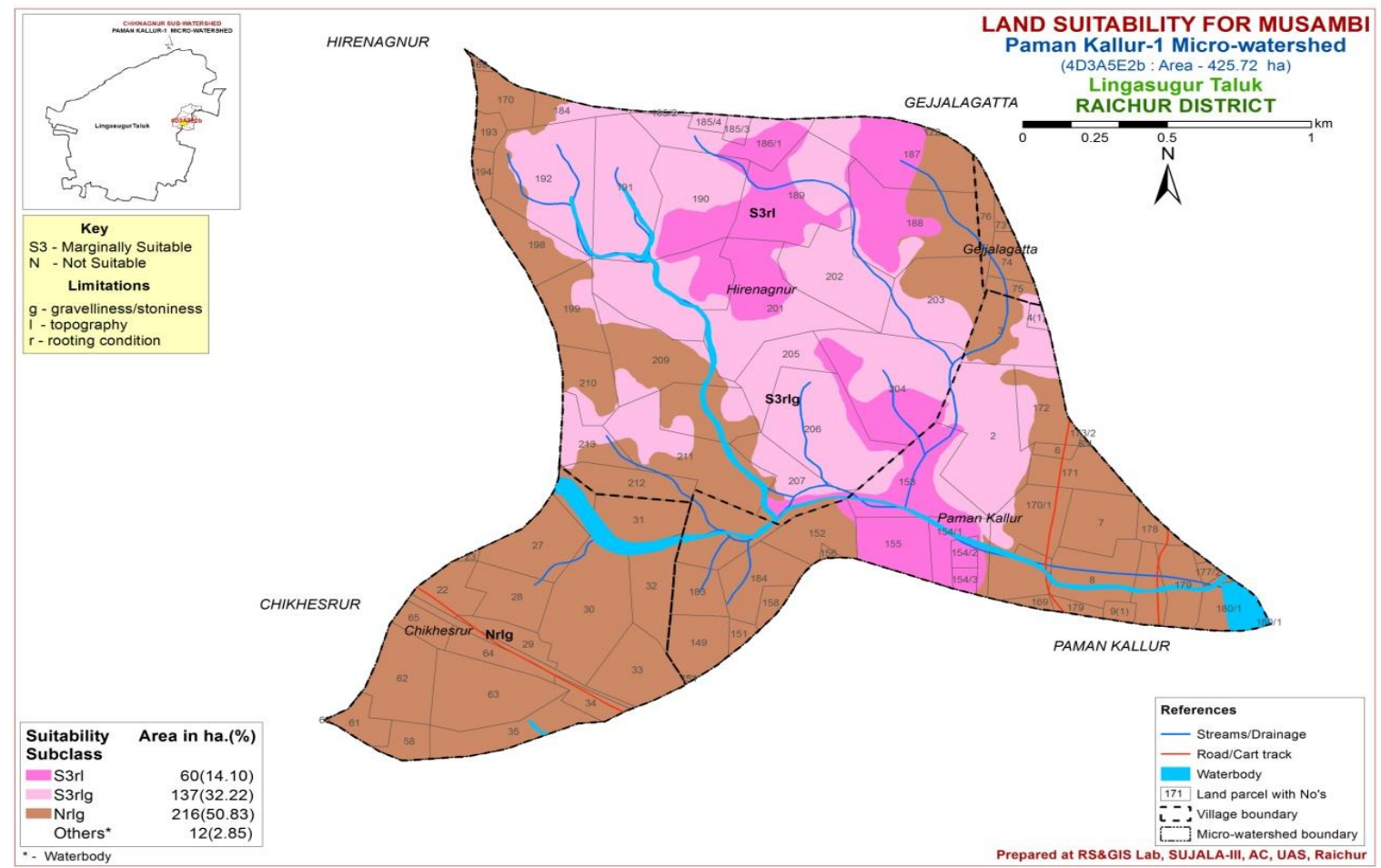

Fig.16 Land suitability map for Tamarind in Paman kallur-1 micro-watershed

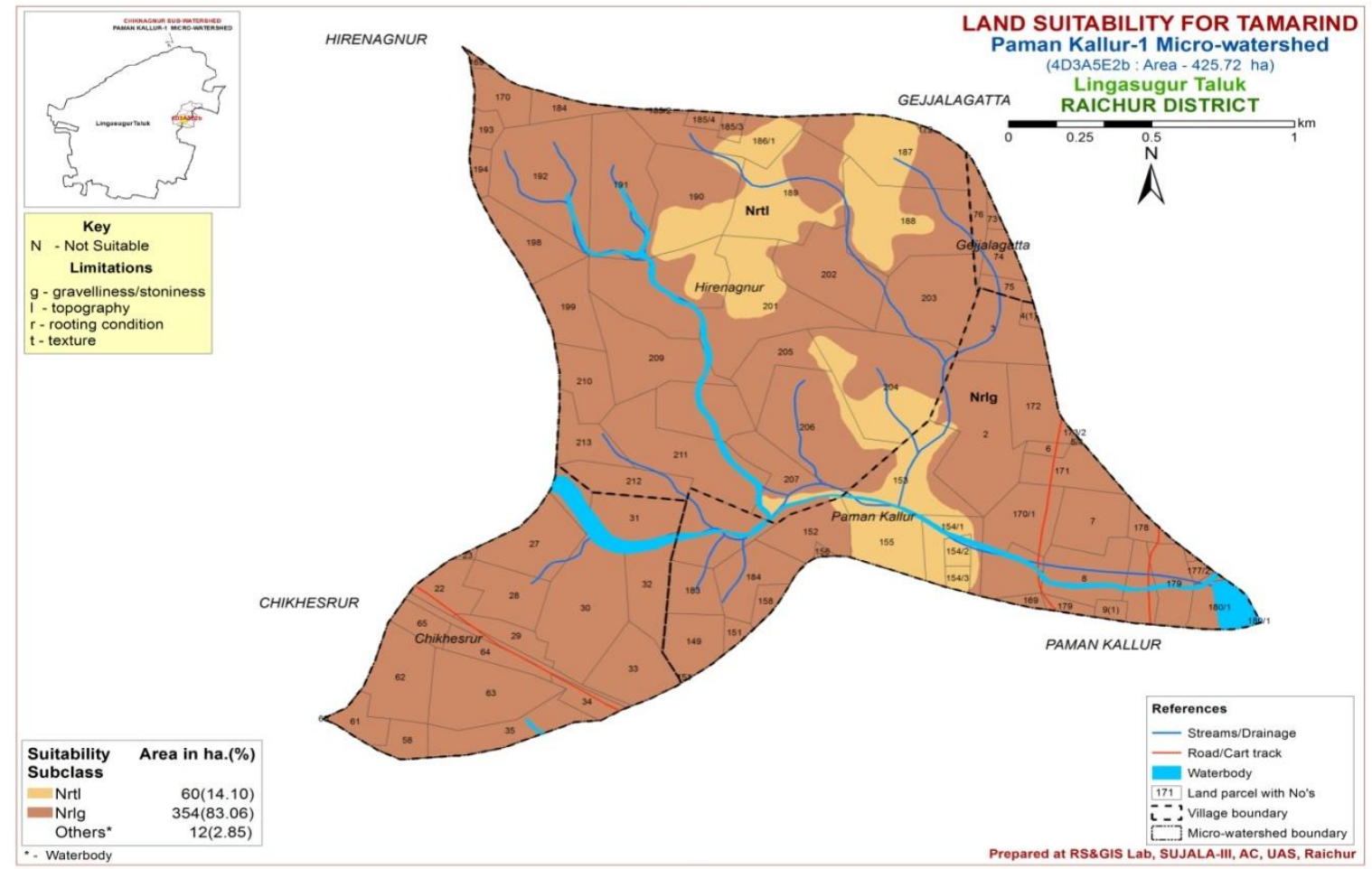


Fig.17 Land suitability map for Jackfruit in Paman kallur-1 micro-watershed

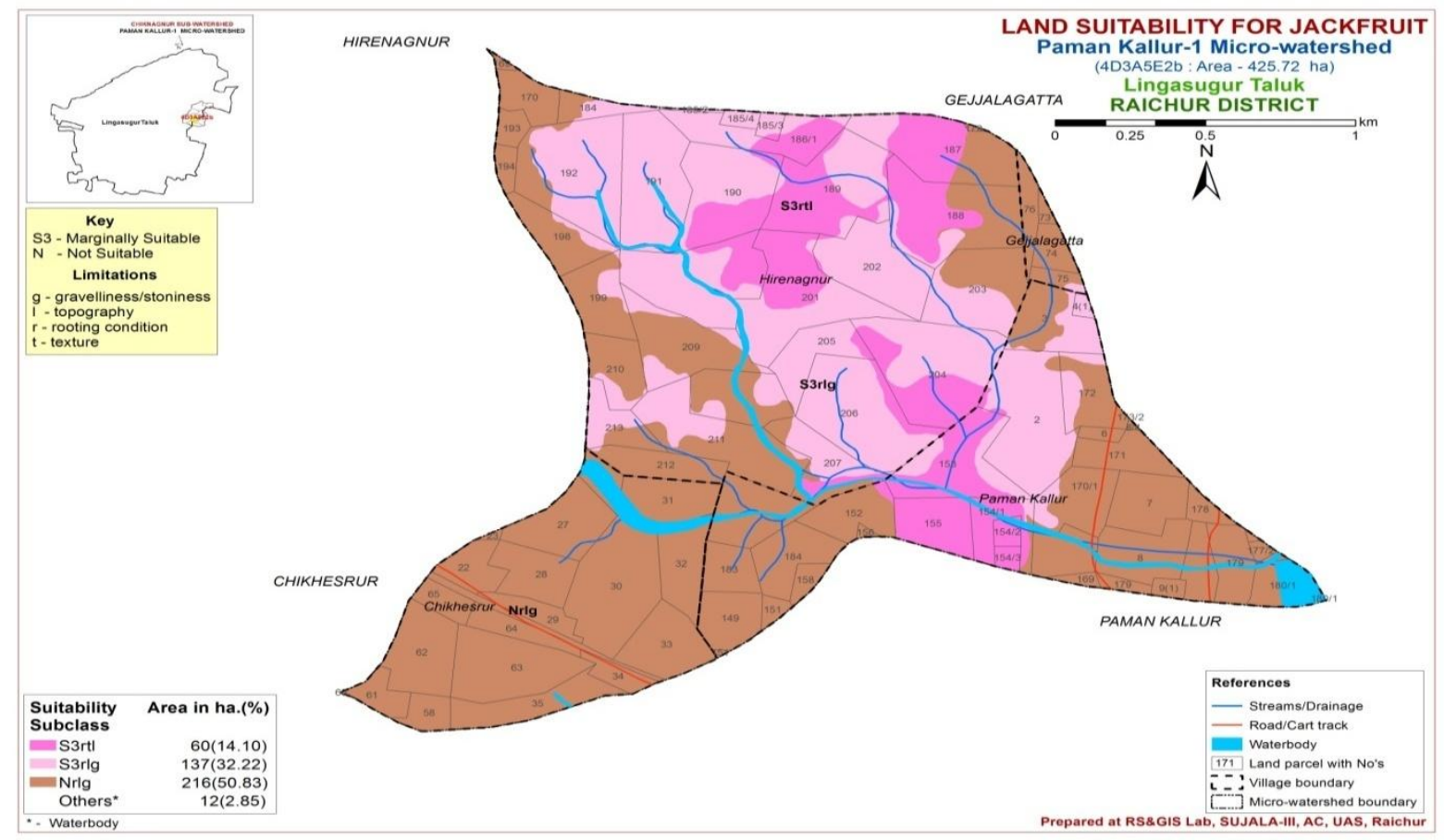

Fig.18 Land suitability map for Pomegranate in Paman kallur-1 micro-watershed

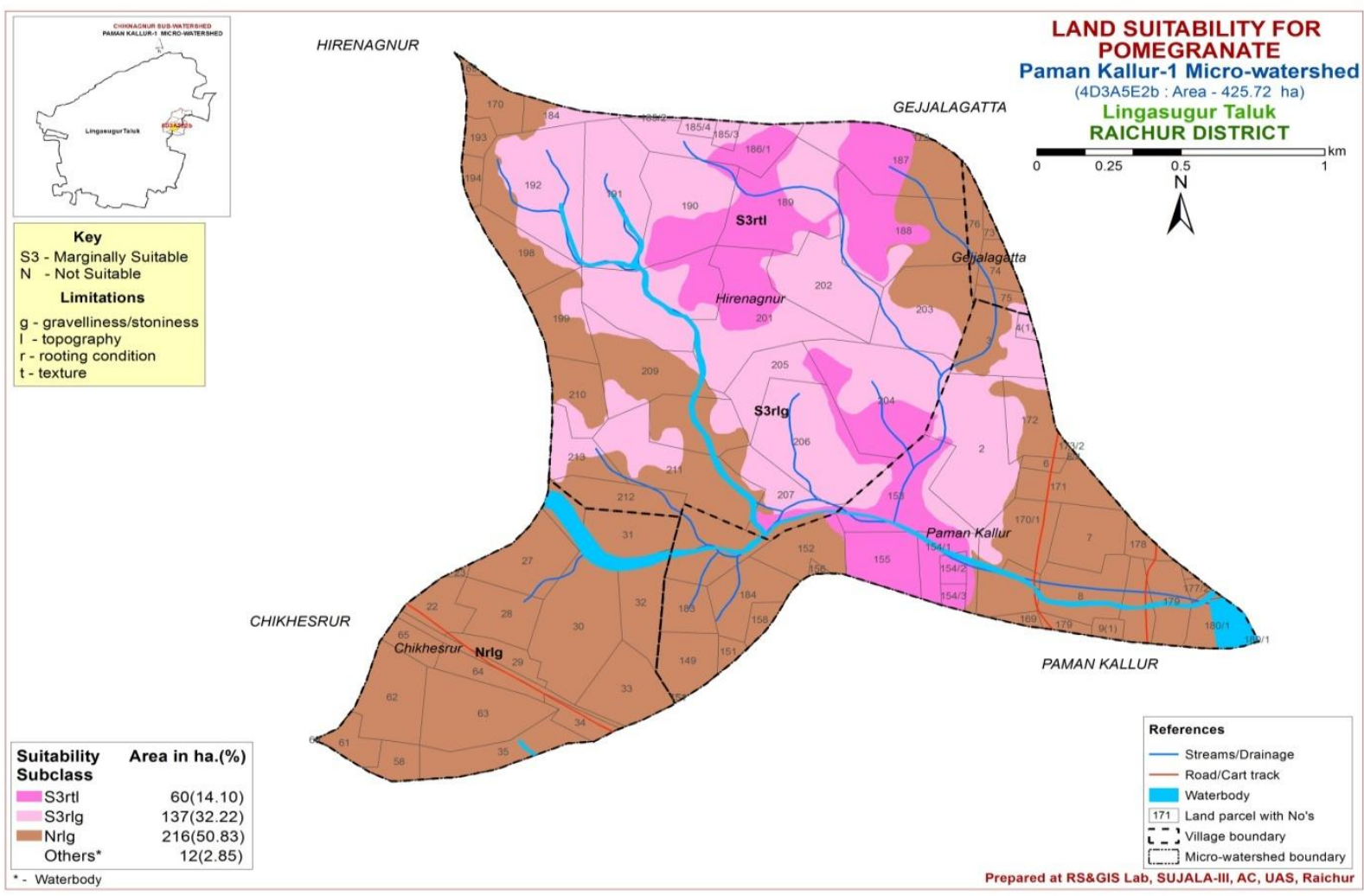


Whereas, CHRhC2g1S1, CHRhD2g1S1R1, CHRhE2g1S1R1, KMThC2g1S1 and THDhD2g1S1R1 soil phase units has interventions like deep and wider size pit for fruit crops, drip irrigation with suitable soil and water conservation measures and cultivation on raised beds with mulches and drip irrigation for vegetables (Table 4).

\section{References}

Asma Najafiana, Mahmood Dayani, Hamid Reza Motaghianc and Habibolah Nadiana. 2012, Geostatistical Assessment of the Spatial Distribution of Some Chemical Properties in Calcareous Soils. J. Integrative Agric., 1729-1737.

Dent, D. and A. Young. 1981. Soil survey and land evaluation. George Allen \& Unwin, London.

Geetha, G. P., Veerendra Patel, G. M., Shruti, Y., Ramakrishna Parama, V. R. and Sathish, A., 2019, Land evaluation in halayapura1 micro watershed in Tumkur district of Karnataka, India, using remote sensing and geographical information system (GIS) tools. Int. J. Chem. Studies., 7(1): 84-94.

Kharche. V. K. and Gaikawad. S. T., 1991, An appraisal of production potentials of suiis of Saongi watershed near Nagpur. Maharashtra. Agrope Joln R., 3: 69-78.

Leelavathi, G. P., Naidu, M. V. S., Ramavatharam, N. and Karuna Sagar,
G., 2009, Studies on genesis, classification and evaluation of soils for sustainable land use planning in Yerpedu mandal of Chittor district, Andra Pradesh. J. Indian Soc. Soil Sci., (57): 109-120.

Naidu, L. G. K., Ramamutrhy, V., Challa, O., Rajendra Hegde. and Krishnan, P.,2006, Manual soil site criteria for major crops. NBSS publication no. 129, NBSS \& LUP, Nagpur, 118.

Rajesh, N. L., Satishkumar, U., Shankergouda, I., Bhat, S. N., Basavaraj, K. and Rudramurthy, H. V., 2018, Detailed LRI for Assessment of Land Capability and Land Suitability of Amarapura-2 Micro-Watershed Using RS and GIS. Int. J. Curr. Microbiol. App. Sci., 7(10): 2370-2381.

Salviano, A. A. C., 1996, Variabilidade de atributos de soloe crotalária júncea em solo degradado do município de Piracicaba-SP. Piracicaba: Tese (Doutorado), Escola Superior de Agricultura Luiz de Queiroz, Universidade de São Paulo, 91.

Sehgal JL. Pedology - Concepts and applications.1996, Kalyani Publishers, New Delhi, 488.

Soil Survey Division Staff, 1999, Soil Survey Manual USDA, Handbook. No. 18 US Government printing office: Washington, DC,

\section{How to cite this article:}

Rajesh, N. L., Kirana Kumara, V. Rajesh, Chandralekha, Ambika Bhandari, S. Deepika, Basavajyoti, Ambika, H. V. Rudramurthy, K. Basavaraj, U. Satishkumar and Desai, B. K. 2019. Land Suitability Evaluation for Legume Crops and Horticulture Crops in Paman Kallur-1 Micro-Watershed using Geospatial Techniques. Int.J.Curr.Microbiol.App.Sci. 8(12): 13021317. doi: https://doi.org/10.20546/ijcmas.2019.812.159 